\title{
Article
}

\section{Cell Surface Expression of Nrg1 Protein in Candida auris}

\author{
Anuja Paudyal and Govindsamy Vediyappan *(i) \\ Division of Biology, Kansas State University, Manhattan, KS 66506, USA; anuja@ksu.edu \\ * Correspondence: gvediyap@ksu.edu
}

Citation: Paudyal, A.; Vediyappan, G. Cell Surface Expression of Nrg1 Protein in Candida auris. J. Fungi 2021, 7, 262. https://doi.org/10.3390/ jof7040262

Academic Editors: Jacques F. Meis and Anuradha Chowdhary

Received: 17 February 2021

Accepted: 28 March 2021

Published: 31 March 2021

Publisher's Note: MDPI stays neutral with regard to jurisdictional claims in published maps and institutional affiliations.

Copyright: (c) 2021 by the authors. Licensee MDPI, Basel, Switzerland. This article is an open access article distributed under the terms and conditions of the Creative Commons Attribution (CC BY) license (https:// creativecommons.org/licenses/by/ $4.0 /)$.

\begin{abstract}
Candida auris is an emerging antifungal resistant human fungal pathogen increasingly reported in healthcare facilities. It persists in hospital environments, and on skin surfaces, and can form biofilms readily. Here, we investigated the cell surface proteins from $C$. auris biofilms grown in a synthetic sweat medium mimicking human skin conditions. Cell surface proteins from both biofilm and planktonic control cells were extracted with a buffer containing $\beta$-mercaptoethanol and resolved by 2-D gel electrophoresis. Some of the differentially expressed proteins were excised and identified by mass spectrometry. C. albicans orthologs Spe3p, Tdh3p, Sod2p, Ywp1p, and Mdh1p were overexpressed in biofilm cells when compared to the planktonic cells of $C$. auris. Interestingly, several proteins with zinc ion binding activity were detected. Nrg1p is a zinc-binding transcription factor that negatively regulates hyphal growth in C. albicans. C. auris does not produce true hypha under standard in vitro growth conditions, and the role of Nrg1p in C. auris is currently unknown. Western blot analyses of cell surface and cytosolic proteins of C. auris against anti-CalNrg1 antibody revealed the Nrg1p in both locations. Cell surface localization of Nrg1p in C. auris, an unexpected finding, was further confirmed by immunofluorescence microscopy. Nrg1p expression is uniform across all four clades of $C$. auris and is dependent on growth conditions. Taken together, the data indicate that $C$. auris produces several unique proteins during its biofilm growth, which may assist in the skin-colonizing lifestyle of the fungus during its pathogenesis.
\end{abstract}

Keywords: Candida auris biofilm; drug-resistant; sweat medium; cell surface proteins; BME extract; 2-DE; Zn-finger protein; cell surface-associated Nrg1; anti-Nrg1 antibody; immunofluorescence

\section{Introduction}

Skin, a major interface between host and environment, is a habitat for several microbial populations. Many skin residing microbes have the ability to form skin biofilms that can lead to skin infection, and in certain conditions, can enter the body to cause life threatening bloodstream infections [1].

Candida auris is an emerging multi-drug resistant human fungal pathogen detected in many countries simultaneously [2]. It colonizes skin surfaces and can be invasive in immunocompromised patients [3]. C. auris has been isolated from blood, wounds, body fluids, and skin [4], where bloodstream infection is the most common infection leading to a mortality rate as high as 30-60\% [5]. Additionally, the ability of C. auris to adhere to and to form drug-resistant biofilms has been a serious issue [6,7]. Until recently, $C$. albicans has been considered the major pathogen but recently, there has been increasing evidence showing $C$. auris involved in biofilm formation resulting in fatal bloodstream infections [8]. Unlike C. albicans, C. auris does not produce hyphae under in vitro conditions but it can form hyphae in vivo [9] or elongated pseudo hyphae in the presence of genotoxic compounds [10].

Nrg1p is a zinc-finger domain containing DNA-binding protein and is a key transcription factor that negatively regulates hyphal growth in C. albicans [11,12]. The NRG1 transcript is down-regulated during hypha-inducing growth conditions, and its deletion has caused constitutive filamentous growth, similar to the tup1 null mutant under yeastpromoting growth conditions because the expression of hypha-specific genes (e.g., UME6, 
ECE1, HWP1, etc.) was derepressed [12]. In a transcriptomic study, hundreds of genes were found to be differentially regulated in a NRG1-dependent and NRG1-TUP1-dependent manner. Studies have also shown that C. albicans Nrg1p plays important roles in biofilm formation and dispersion, and deletion or overexpression of NRG1 blocks the yeast-tohypha transition resulting in attenuated virulence [12-14]. However, the expression of $C$. auris NRG1 and its role in virulence is unknown.

Sweat media that mimics axillary skin condition promotes $C$. auris biofilm formation in vitro and biofilm produced by $C$. auris in this media was ten times more robust than that of biofilms formed by C. albicans. According to a recent study, high salinity and fatty acids mimicking sweat favors growth of $C$. auris allowing it to survive for 14 days whereas C. albicans survived for less than a week [15]. Since cell surface proteins play a major role in host cell adherence and biofilm formation, understanding their expression and their cellular roles in the pathogenicity of $C$. auris will help develop effective intervention strategies. We used sweat medium to study C. auris biofilm proteins.

In this study, we extracted non-glucan attached cell surface proteins from C. auris biofilm and planktonic cells, separated by 2-D gel electrophoresis, and identified some of the differentially expressed proteins by liquid chromatography coupled with mass spectrometry. We have also demonstrated an unexpected finding of cell surface localization of C. auris Nrg1p, which is a zinc-finger transcription factor and is predicted to be nuclear.

\section{Materials and Methods}

\subsection{C. auris Strains and Growth Conditions}

Candida species were routinely maintained on YPD (1\% yeast extract, $2 \%$ peptone, and $2 \%$ dextrose) agar (1.5\%). Candida auris isolated from Saudi Arabia by Abdalhamid et al. [16] (South Asian, clade-I) was used for most of the experiments in this study. A single colony of this $C$. auris was inoculated into YPD broth medium and grown overnight at $37^{\circ} \mathrm{C}$ with shaking at $200 \mathrm{rpm}$. For planktonic cells, overnight grown cells were used to inoculate $100 \mathrm{~mL}$ of fresh sweat medium to make a suspension of approximately $2 \times 10^{5}$ cells at an optical density of $0.05\left(\mathrm{OD}_{600}\right)$ and grown at $37^{\circ} \mathrm{C}$ for $48 \mathrm{~h}$ with shaking (200 rpm). Sweat medium was prepared as mentioned by Horton et al. [15]. Biofilms were formed in cell culture treated dishes using the same cell density as for planktonic growth. They were allowed to grow at $37^{\circ} \mathrm{C}$ for $48 \mathrm{~h}$ without disturbance. For pseudohyphal induction by hydroxyurea (HU) in C. auris [10], the sweat medium was used with and without $100 \mathrm{mM} \mathrm{HU}$ and grown statically for $48 \mathrm{~h}$ at $37^{\circ} \mathrm{C}$. C. auris strains (Table 1) (Clades II-IV), representing those from different countries, were obtained from the Centers for Disease Control and Prevention (CDC) and the Food and Drug Administration (FDA) Antibiotic Resistance (AR) isolate bank (Atlanta, GA, USA).

Table 1. List of Candida fungal strains used in this study.

\begin{tabular}{cc}
\hline Strains & Source \\
\hline Candidaauris (Lab strain \#1126, Clade I) & Abdalhamid et. al. (2018) [16] \\
\hline C. auris (AR\#0381, Clade II) & $\begin{array}{c}\text { Antibiotic Resistance (AR) Isolate Bank, the } \\
\text { Centers for Disease Control and Prevention (CDC) } \\
\text { and the Food and Drug Administration (FDA) }\end{array}$ \\
\hline C. auris (AR\#0383, Clade III) & AR Isolate Bank, CDC/FDA \\
\hline C. auris (AR\#0386, Clade IV) & AR Isolate Bank, CDC/FDA \\
\hline C. albicans (Lab strain \#1) & Wildtype (SC5314) [17] \\
\hline C. albicans $n r g 1^{-/-}$ & Noble's deletion mutants [18]
\end{tabular}

\subsection{Cell Surface Proteins Preparation}

Planktonic cells were collected by centrifugation while biofilm cells were scraped from cell culture dishes using a cell scraper. Fungal cells were washed twice with sterile 
phosphate buffered saline (PBS), $\mathrm{pH}$ 7.5, to remove residual media components. Cell surface proteins (non-glucan bound) were extracted from both planktonic and biofilm cells by resuspending them in ammonium carbonate $(1.89 \mathrm{~g} / \mathrm{L})$ buffer containing $1 \% \beta$ mercaptoethanol (BME) and incubated at $37^{\circ} \mathrm{C}$ for $30 \mathrm{~min}$ as described $[19,20]$. Pooled BME extracts were dialyzed thoroughly against water and lyophilized before analyses.

\subsection{2-Dimensional Gel Electrophoresis (2-DE)}

Lyophilized cell surface proteins were resuspended in a small volume of sterile water and their concentration determined using the Bradford dye-binding method [21]. 2-DE was performed using the immobilized $\mathrm{pH}$ gradient (IPG) gels (Immobiline Drystrip, $\mathrm{pH}$ $3-10$, NL, $7 \mathrm{~cm}$, Cytiva, MA, USA). Aliquots of protein suspensions were diluted in IPG buffer as described by the manufacturer and applied to IPG gels. The gel strips were rehydrated overnight at room temperature. First dimension electrofocusing was performed using Multiphore-II unit (Pharmacia Biotech, Uppsala, Sweden) at $20^{\circ} \mathrm{C}, 3500 \mathrm{~V}$ for $6 \mathrm{~h}$. Gels were equilibrated first in an equilibration buffer containing dithiothreitol for $15 \mathrm{~min}$, then in a buffer containing iodoacetamide for another $15 \mathrm{~min}$ as recommended by the manufacturer. Electrofocused gel strips were run in the second dimension using $12.5 \%$ SDSPAGE for $90 \mathrm{~min}$ at $100 \mathrm{~V}$. Resolved proteins were stained by silver stain (Sigma-Aldrich, St. Louis, MO, USA) and images were recorded against a white light background. Cell surface proteins from at least two different biological samples were analyzed by 2-DE. Proteins that were differentially expressed were identified and some were excised for LC-MS/MS analysis. When necessary, 2-DE separated proteins were transferred to an Immobilon-P polyvinylidene fluoride (PVDF) membrane (Millipore Sigma, Billerica, MA, USA) electrophoretically for Western analysis.

\subsection{Mass Spectrometry Analysis}

Proteins from 2-DE were analyzed by a core facility service (DNA/Protein Resource Facility, Oklahoma State University, Stillwater, OK, USA). Proteins from the excised gel spots were digested with trypsin and collected. Peptides were dissolved in $0.1 \%$ formic acid, and injected onto a 75-micron $\times 55 \mathrm{~cm}$ nano HPLC column packed with 2-micron C18 media (Thermo Fisher PN 164942). Peptides were separated using $0.1 \%$ aqueous formic acid as mobile phase A and acetonitrile/water/formic acid (80:20:0.1) as mobile phase B to develop a gradient of $0-35 \%$ over $60 \mathrm{~min}$. The column was terminated with stainless-steel emitter within a Nanospray Flex Ion source (Thermo Fisher, Waltham, MA, USA). The ion stream was analyzed in a quadrupole-Orbitrap tribrid mass spectrometer (Orbitrap Fusion model, Thermo Fisher, Waltham, MA, USA), using a data-dependent 5-s Top Speed method, wherein peptide precursors were measured in the Orbitrap sector, isolated in the quadrupole sector and fragmented in the ion routing multipole. After dissociation, peptide fragment ions were analyzed in the ion trap sector. The results were analyzed using MaxQuant software and the C. auris B8441 reference genome. Criteria such as peptide ion signal peak intensity, peptide spectral match (PSM)-based quantity, $Q$ values, peptide mass, $\mathrm{pI}$, and sequence coverage were included during analysis.

\subsection{Bioinformatic Analyses}

The amino acid sequence of Nrg1p from C. albicans (C7_04230W_A, Candida Genome Database, CGD) was blasted (NCBI blast) against that of C. auris (B9J08_005429, B8441, CGD) to determine the percent homology between them. Iterative Threading ASSEmbly Refinement (I-TASSER) [22] was used to generate a 3D model of $C$. auris and C. albicans $\mathrm{Nrg} 1$ protein based on previously available 3D structures of one or more closely related proteins. Using 3D models from I-TASSER, structural alignment of $C$. auris and C. albicans Nrg1 was carried out using RaptorX structural alignment tool [23]. 


\subsection{NRG1 Cloning, Nrg1p Purification and Antibody Production}

The gene (NRG1) encoding Nrg1p of C. albicans was synthesized and cloned in-frame in an Escherichia coli overexpression vector (pET24a, Kan ${ }^{\mathrm{R}}, \mathrm{NdeI}$ and XhoI, Epoch Life Sciences Inc., Missouri City, TX, USA) that contains a 6-Histidine tag at its C-terminus. The plasmid was transformed into E. coli (BL21-DE3 or Rosetta) cells. Nrg1p was overexpressed by induction with $1 \mathrm{mM}$ of isopropyl- $\beta$-d-1-thiogalactopyranoside (IPTG) in antibiotic containing medium. Expressed cells were pelleted and resuspended in phosphate buffer containing a protease inhibitors cocktail followed by cell lysis using a French press $(19,000 \mathrm{psi})$ with centrifugation at $10,000 \times \mathrm{g}$ for $10 \mathrm{~min}$ at $4{ }^{\circ} \mathrm{C}$. Nrg1p from the supernatant was purified using nickel-nitrilotriacetic acid (Ni-NTA) coupled agarose beads (Qiagen, Germantown, MD, USA) as recommended by the manufacturer. Thus, the purified Nrg1p was dialyzed against water, verified for homogeneity on SDS-PAGE and used for antibody production (CalNrg1 antibody) in a rabbit (LAMPIRE Biological Laboratories, Pipersville, PA, USA).

\subsection{Immunoblotting}

CalNrg1 polyclonal antibody was used to detect C. auris Nrg1p. Cell surface and cytosolic proteins from both planktonic and biofilm cells were separated by SDS-PAGE after boiling and reducing conditions and transferred to an Immobilon-P PVDF membrane (Millipore Sigma, Billerica, MA, USA). The membrane was blocked using 5\% non-fat dry milk in a buffer containing Tris- $\mathrm{HCl}, \mathrm{NaCl}$, and Tween-20 and blotted against an antirabbit CalNrg1 antibody followed by an anti-rabbit IgG-HRP conjugate (R \& D System, Minneapolis, MN, USA). The ECL Western Blotting Substrate (Thermo Scientific, Waltham, MA, USA) was used to detect the protein bands followed by imaging in Azure c600.

\subsection{Immunofluorescence}

C. auris cells grown in sweat medium for $48 \mathrm{~h}$ at $37^{\circ} \mathrm{C}$ were collected and washed twice using PBS to remove media constituents. Immunofluorescence of $C$. auris after immunostaining was performed as described [24] with slight modifications. Briefly, cells were fixed in $4 \%$ formaldehyde for $15 \mathrm{~min}$ followed by a PBS wash. Fixed cells were then blocked with 3\% BSA in PBS containing 0.1\% Tween-20, washed with PBS and incubated with 1:100 anti-CalNrg1 antibody raised in a rabbit overnight at $4{ }^{\circ} \mathrm{C}$. Cells were washed twice with PBS and further incubated in an anti-rabbit Alexa Fluor-secondary antibody (Thermo Fisher Scientific, Waltham, MA, USA) 1:100 for $2 \mathrm{~h}$. Cells were washed three times and mounted on a slide to examine under a fluorescence microscope (Leica DM 6 B with a camera attached).

\section{Results}

\subsection{Cell Surface Proteins Are Differentially Expressed in Planktonic and Biofilm Cells}

Cell surface proteins play a major role in adhesion and biofilm formation. To identify the differentially expressed non-glucan attached cell surface proteins from biofilm and planktonic C. auris cells (strain \#1126, Clade I, Table 1), we used proteomics approaches. Using 2-D gel electrophoresis followed by silver staining, several differentially expressed proteins can be found and representative images are shown (Figure 1a,b). Selected protein spots from the biofilm sample were excised from the gel and analyzed by mass spectrometry. These cell surface proteins are listed in Table 2. C. albicans ortholog proteins including Spe3p, Tdh3p, Sod2p, Ywp1p and Mdh1p were present in high intensities in C. auris biofilm cells when compared to planktonic cells. 


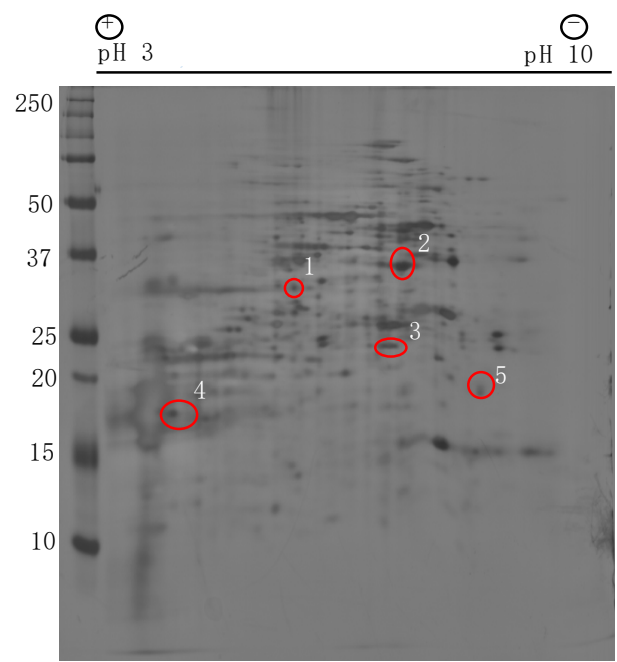

(a)

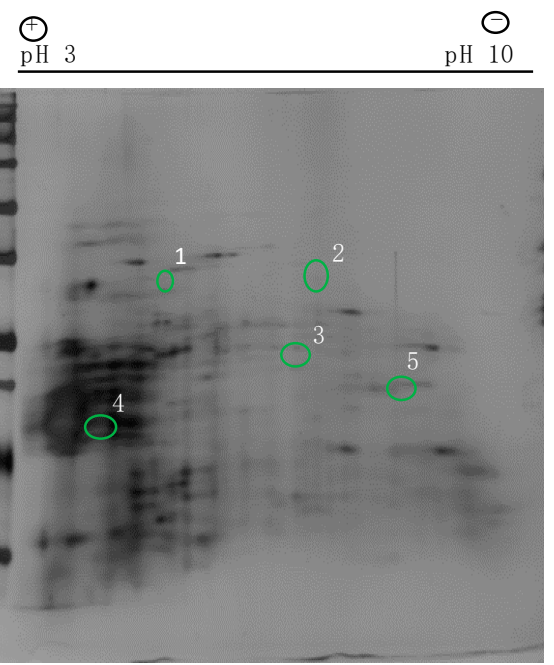

(b)

Figure 1. Cell surface proteins extracted from Candida auris biofilm and planktonic cells and resolved by 2-Dimensional Gel Electrophoresis (2-DE). Silver stained gel images of proteins from biofilm (a) and planktonic cells (b). Protein spots marked with red circles (a) are excised out and identified by mass spectrometry. These proteins were expressed less or were absent in planktonic cells (marked in green circles) (b).

Table 2. List of proteins identified by MS. Proteins are selected on the basis of their peptide ion signal intensity, matching peptide and sequence coverage. $Q$-value of zero applies to all identified proteins.

\begin{tabular}{|c|c|c|c|c|c|c|c|c|c|c|c|}
\hline \multirow{2}{*}{$\begin{array}{l}\text { Spot } \\
\text { no. }\end{array}$} & \multirow{2}{*}{$\begin{array}{l}\text { Protein } \\
\text { C. auris }\end{array}$} & \multirow{2}{*}{ Intensity } & \multirow{2}{*}{$\begin{array}{l}\text { Number } \\
\text { of } \\
\text { Peptide }\end{array}$} & \multirow{2}{*}{$\begin{array}{c}\text { Seq } \\
\text { Coverage } \\
(\%)\end{array}$} & \multicolumn{2}{|c|}{ Molecular Weight (kDa) } & \multicolumn{2}{|c|}{$\mathrm{pH}$} & \multirow{2}{*}{ Localization } & \multirow{2}{*}{ Function } & \multirow{2}{*}{$\begin{array}{c}\text { C. } \\
\text { albicans } \\
\text { Ortholog }\end{array}$} \\
\hline & & & & & Predicted & Experimental & Predicted & Experimental & & & \\
\hline 1 & A0A2H0ZC50 & $85,544,000$ & 8 & 18.3 & 33.618 & $25-37$ & 5.12 & 5.9 & $\begin{array}{l}\text { Extracellular } \\
\text { region }\end{array}$ & $\begin{array}{l}\text { Spermidine } \\
\text { synthase }\end{array}$ & Spe3p \\
\hline 2 & A0A2H1A5V9 & $131,870,000$ & 20 & 66.2 & 34.541 & 37 & 6.81 & 6.5 & $\begin{array}{l}\text { Extracellular } \\
\text { region, } \\
\text { mitochon- } \\
\text { dria }\end{array}$ & $\begin{array}{c}\text { Malate } \\
\text { dehydroge- } \\
\text { nase } \\
\text { precursor }\end{array}$ & Mdh1p \\
\hline 3 & A0A2H1A3N7 & $8,158,000$ & 10 & 42.2 & 25.404 & 25 & 7.16 & 6.5 & $\begin{array}{l}\text { Cytoplasm, } \\
\text { mitochon- } \\
\text { dria }\end{array}$ & $\begin{array}{l}\text { Superoxide } \\
\text { dismutase }\end{array}$ & Sod2p \\
\hline 4 & A0A2H0ZMT8 & 305,020 & 3 & 7 & 46.13 & $15-20$ & 4.65 & 5 & $\begin{array}{c}\text { Cell } \\
\text { surface, } \\
\text { cell wall }\end{array}$ & $\begin{array}{l}\text { Yeast form } \\
\text { well protein }\end{array}$ & Ywp1p \\
\hline 5 & A0A2H0ZM53 & $46,601,000$ & 9 & 29.3 & 35.336 & 20 & 7.13 & 7.3 & $\begin{array}{l}\text { Cytoplasm, } \\
\text { cell wall, } \\
\text { cell } \\
\text { surface }\end{array}$ & $\begin{array}{l}\text { Glyceraldehyde } \\
\text { 3-phosphate } \\
\text { dehydroge- } \\
\text { nase } \\
\text { activity }\end{array}$ & Tdh3 \\
\hline
\end{tabular}

Protein spot \#1 is spermidine synthase (Spe3p). It localizes in the extracellular region and plays role in spermidine and pantothenate biosynthesis. Similarly, Tdh3p was also expressed more in the biofilm cells which are known to be located in the cytoplasm, cell wall and cell surface regions. An increased TDH3 transcript has been reported in $C$. albicans biofilm [25]. The next protein that showed increased expression in biofilm cells was Sod2p (spot \#3) which has superoxide dismutase activity and is known to be found in the mitochondrial matrix [26,27]. Ywp1p is a yeast wall protein-1 found on the cell surface and cell wall of yeasts known to be involved in adhesion and biofilm formation [28]. Mdh1 is a predicted malate dehydrogenase precursor and is found in the extracellular regions and mitochondria. It was upregulated in C. albicans when grown as a biofilm [29]. Future studies are required to identify additional proteins that are differentially expressed and validate them. 


\subsection{Nrg1 Protein Expresses in C. auris Biofilm and Planktonic Cells}

C. albicans is known to cause biofilm-related infections and strains that are defective in hyphal growth fail to produce robust biofilm and matrix polysaccharides [30,31]. Nrg1p is a negative regulator of hyphal growth in C. albicans, but its role and expression of $\mathrm{Nrg} 1$ in $C$. auris is unknown. Since $C$. auris produces yeast cells during in vitro biofilm growth and a gene encoding Nrg1p is present in C. auris (B8441 genome, B9J08_005429, C. albicans ortholog), we were interested in investigating it in C. auris. Further, Nrg1p is a zinc-binding transcription factor and we identified other zinc-binding proteins including Fba1p, Adh1p, Xyl2p and Pmi1p in our MS analysis, prompting us to investigate Nrg1p further.

To determine Nrg1p expression and localization in C. auris, we used an anti-CalNrg1 antibody (polyclonal) that we raised in our laboratory. Briefly, C. albicans NRG1 with 6His tag at the C-terminus was cloned, overexpressed and purified in an E. coli expression system. The purified Nrg1 protein with a molecular weight of approximately $36 \mathrm{kDa}$ reacted to both the anti-His and anti-CalNrg1 antibody (Figure 2a). To examine Nrg1 protein expression in C. auris, it was grown in sweat medium as planktonic and biofilm cells. Using the CalNrg1 antibody, we detected an Nrg1 protein band of $\sim 52 \mathrm{kDa}$ in both the C. auris cell surface and cytosolic protein extracts of biofilm and planktonic cells (Figure $2 b$ ). The predicted molecular weight of C. auris Nrg1p is $26.1 \mathrm{kDa}$ (B9J08_005429) and the $52 \mathrm{kDa}$ reactive band is likely to be a dimer of Nrg1p. Nrg1p is expressed slightly more in the cell surface proteins of biofilm than that of planktonic cells (Figure 2b) even though an equal amount of protein was used (Ponceau staining, data not shown). The antibody reacted to C. albicans cytosolic Nrg1 protein but none in the cell surface proteins (data not shown). The CalNrg1 antibody did not react to the cytosolic proteins of C. albicans $n r g 1^{-/-}$ (null) mutant [18], consistent with its specificity for Nrg1p (Figure 2b). Anti-GAPDH (glyceraldehyde 3-phosphate dehydrogenase)antibody (human, horseradish peroxidase (HRP)-conjugated, Santa Cruz Biotechnology, TX, USA) reacted with a cytosol protein component but not with a cell surface protein component.

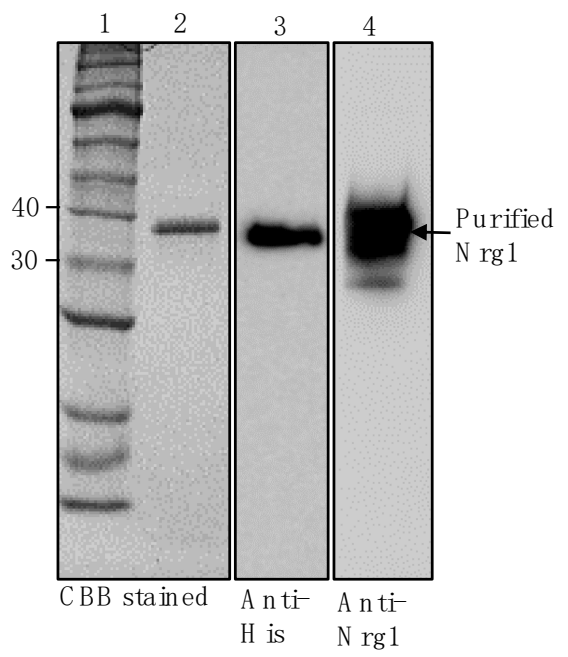

(a)

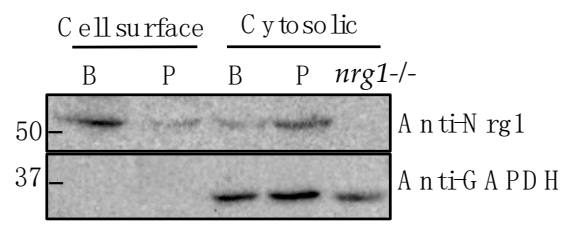

(b)

Figure 2. Purification of C. albicans Nrg1 protein overexpressed in Escherichia coli and the expression of Nrg1p in C. auris biofilm and planktonic cells. (a) Purified rCalNrg1-6His protein separated on SDSPAGE. Lane 1. Protein ladder (New England Biolab, Boston, MA, USA), Lane 2. $\mathrm{Ni}^{++}$affinity purified CalNrg1p (Coomassie blue stained), Lane 3. Western blot of purified CalNrg1p against anti-His antibody, and Lane 4. Western blot of purified CalNrg1p against anti-CalNrg1 antibody. (b) Western blot of cell surface and cytosolic proteins of C. auris biofilm (B) and planktonic (P) cells probed with anti-CalNrg1 antibody. C. albicans $\mathrm{nrg}^{-/-}$was included as negative control. Anti-GAPDH HRP-conjugate provides loading and subcellular location controls. 


\subsection{Nrg1 Proteins of C. albicans and C. auris Are Structurally Similar}

As a transcription factor, $\mathrm{Nrg} 1 \mathrm{p}$ is predicted to be in the nucleus in C. albicans and the cell surface expression of this protein is unexpected. To understand the similarities of $\mathrm{Nrg} 1$ proteins between $C$. auris and C. albicans, we compared their amino acid sequences and aligned their predicted 3D structures using software. C. albicans and C. auris $\mathrm{Nrg} 1$ proteins contain 310 and 236 amino acids with predicted molecular weights of $34.3 \mathrm{kDa}$ and $26.1 \mathrm{kDa}$, respectively. Sequence alignment using Clustal Omega shows a total of $38 \%$ identity (Figure 3a), and Basic Local Alignment Search Tool (BLAST) for protein result shows $67 \%$ identity in the region between 185 to 220 of C. auris and 219 to 254 region of C. albicans $\mathrm{Nrg} 1 \mathrm{p}$. Based on structure prediction software, five structures were predicted for both $C$. auris and C. albicans Nrg1. Structures with the least C-score were picked for both proteins (Figure $3 b, c)$. Using these predicted structures, structural alignment was performed using RaptorX software (Figure 3d) and a template modeling (TM) score of 0.667 was obtained which suggests that these two proteins share a similar fold. Interestingly, the C-terminal $\mathrm{C}_{2} \mathrm{H}_{2}$ zinc-finger domains between these two proteins are highly conserved (Figure 3a-d). The zinc-finger domain is involved in DNA binding activity and this type of Class 1 zinc-finger protein is common in most of the eukaryotes [32].

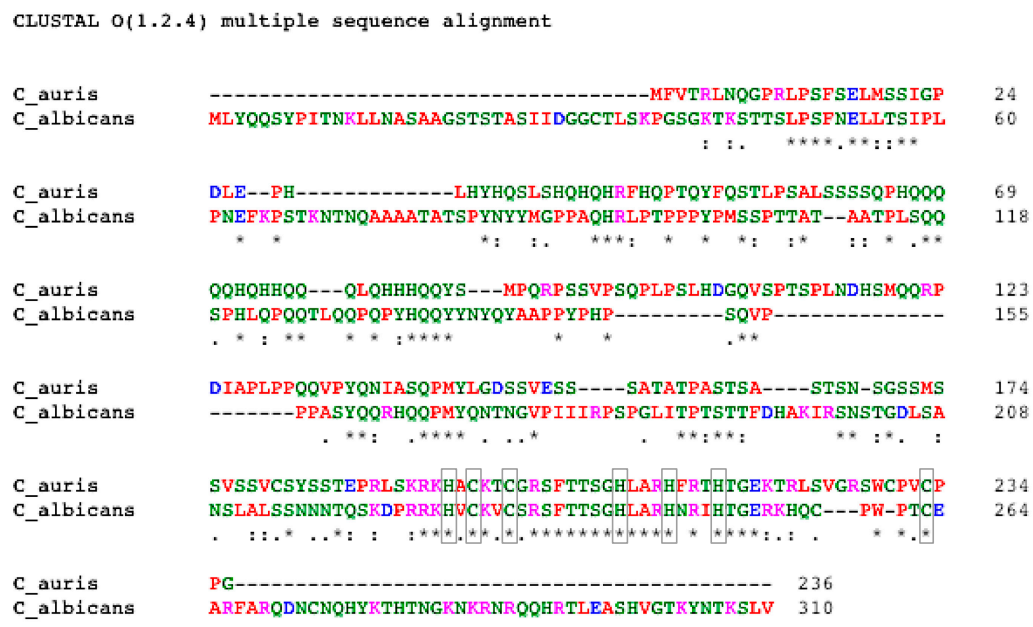

(a)

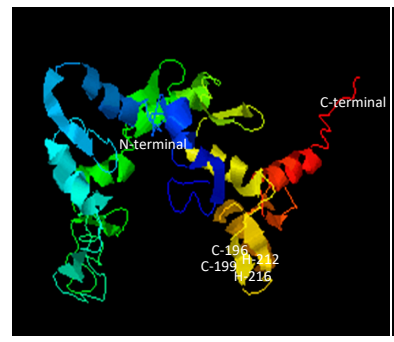

(b)

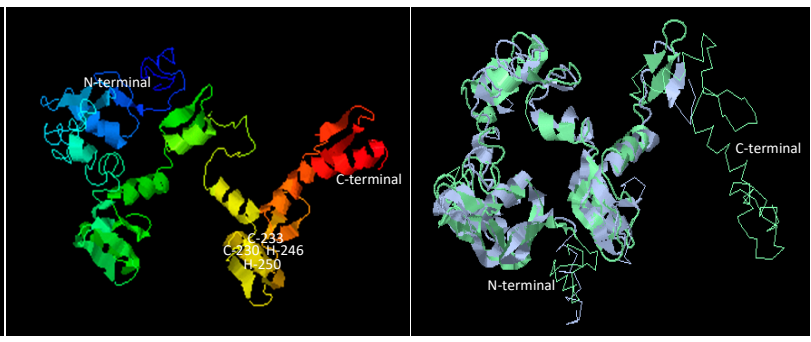

(c) (d)

Figure 3. Comparison between Nrg1p of C. auris and C. albicans. Alignment of amino acid sequences of Nrg1p of C. auris and C. albicans (a). The Cys and His residues $\left(\mathrm{C}_{2} \mathrm{H}_{2}\right.$ zinc-finger) and the identities between the amino acid residues are marked by gray boxes and asterisks, respectively. Predicted 3D structures of $\mathrm{Nrg} 1 \mathrm{p}$ of $\mathrm{C}$. auris (b) and C. albicans (c) using Iterative Threading ASSEmbly Refinement (I-TASSER). N-terminus, C-terminus and predicted zinc-binding domains are marked. (d) Structure alignment of C. auris Nrg1p (purple) and C. albicans Nrg1p (green) using predicted structures from I-TASSER. Note $C$. auris lacks 44 residues after the $\mathrm{C}_{2} \mathrm{H}_{2}$ zinc-finger domain (Class 1 ) at its $\mathrm{C}$-terminus. The aligned image was tilted forward for better viewing, and hence the $\mathrm{N}$-terminal regions appear different from images $(\mathbf{b}, \mathbf{c})$. 


\subsection{Nrg1 Protein Localizes on C. auris Cell Surface}

Having verified bioinformatically the similarities of Nrg1 proteins between C. auris and $C$. albicans and detected the Nrg1p in the cell surface extract of $C$. auris during the 1stdimensional SDS-PAGE/Western analysis (Figures 2 and 3), we next wanted to determine if Nrg1p can be detected from the cell surface fraction of C. auris after 2-DE. This is also because we did not identify Nrg1p in our initial proteomic analyses of $C$. auris biofilm cell surface proteins (2-DE and MS, Figure 1 and Table 2).

We used cell surface proteins (BME extract) collected from C. auris biofilm cells grown in the sweat medium and resolved by 2-DE as above and analyzed by Western blot against the CalNrg1 antibody (Figure $4 \mathrm{~b}$ ). The result shows a single protein spot that reacted to the CalNrg1 antibody that matches the predicted size of $26 \mathrm{kDa}$ of C. auris $\mathrm{Nrg} 1$ protein (Figure $4 \mathrm{~b}$ ). To further validate the specificity of the CalNrg1 antibody to Nrg1 proteins of Candida species, the CalNrg1 antibody was depleted against the purified C. albicans rNrg1-6His protein and used for Western analysis. The depleted antibody failed to detect Nrg1 proteins of $C$. auris cytosolic proteins, suggesting its specificity to Nrg1p (Figure S1).

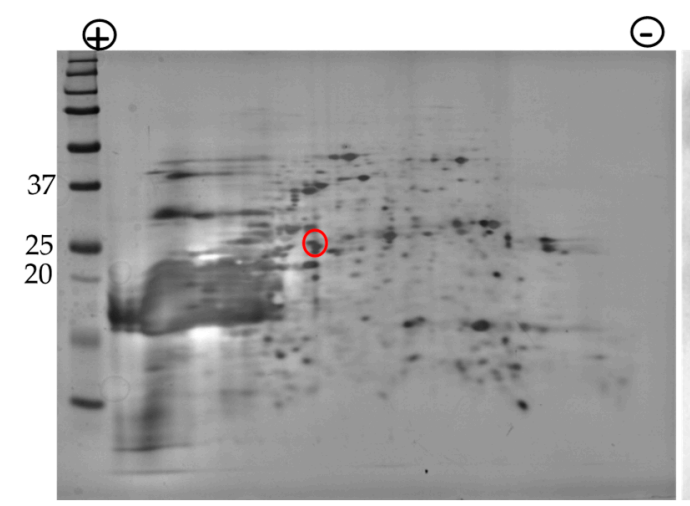

(a)

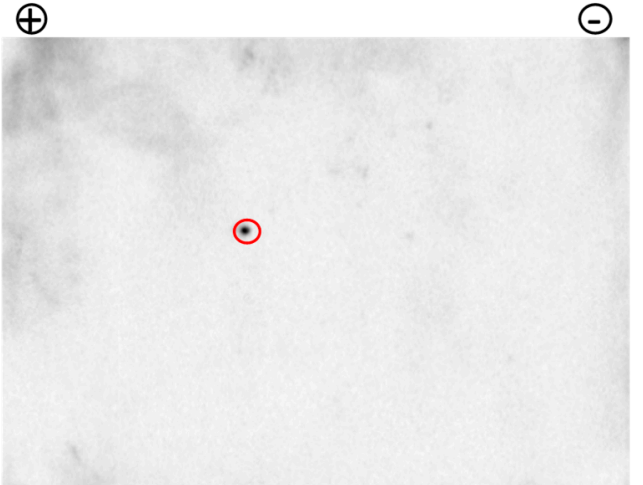

(b)

Figure 4. Nrg1p is localized in the cell surface extract of C. auris. (a), The silver-stained SDS-PAGE gel image of cell surface proteins after 2-DE. (b), Western analysis of the same proteins against rabbit anti-CalNrg1 antibody. The antibody reactive Nrg1p spot (marked in a red circle) with a molecular weight of around $26 \mathrm{kDa}$ is shown in panel (b). All blue prestained protein standard (Bio-Rad, Hercules, CA, USA) was included in the 2nd dimension gel (a). Potential Nrg1p is marked in red circle (a).

To determine the surface localization of the Nrg1p on C. auris cells in vivo, we performed an immunofluorescence assay. C. auris cells were grown in synthetic sweat medium for $48 \mathrm{~h}$ and used CalNrg1 antibody (primary antibody) followed by a fluorescent secondary antibody as described in the Materials and Methods Section.

Interestingly, we observed Nrg1p expression on the surface of $C$. auris cells where several punctate fluorescence signals can be seen (Figure 5). These fluorescence signals were discrete and found throughout the cell surface. Cells stained with a second antibody coupled with Alexa Fluor only (minus the primary antibody) did not show any fluorescent signal (Figure 5, lower panel).

\subsection{Nrg1 Protein Expresses in Different Clades of C. auris and Is Dependent on Growth Conditions}

To know if Nrg1p expresses in different clades of C. auris, we obtained a panel of C. auris strains (Clades II-IV) from the CDC and used one strain from each clade along with C. auris strain \#1126 (Clade I) to analyze Nrg1p expression. Cells grown in the sweat medium were homogenized and the cytosolic proteins were used for Western analysis. Results shown in Figure 6a indicate Nrg1p expresses in all the four strains representing different clades. We also determined the expression of Nrg1p in a rich medium, YPD broth, 
and found it was poorly expressed in this medium when compared to the sweat medium (Figure 6b).
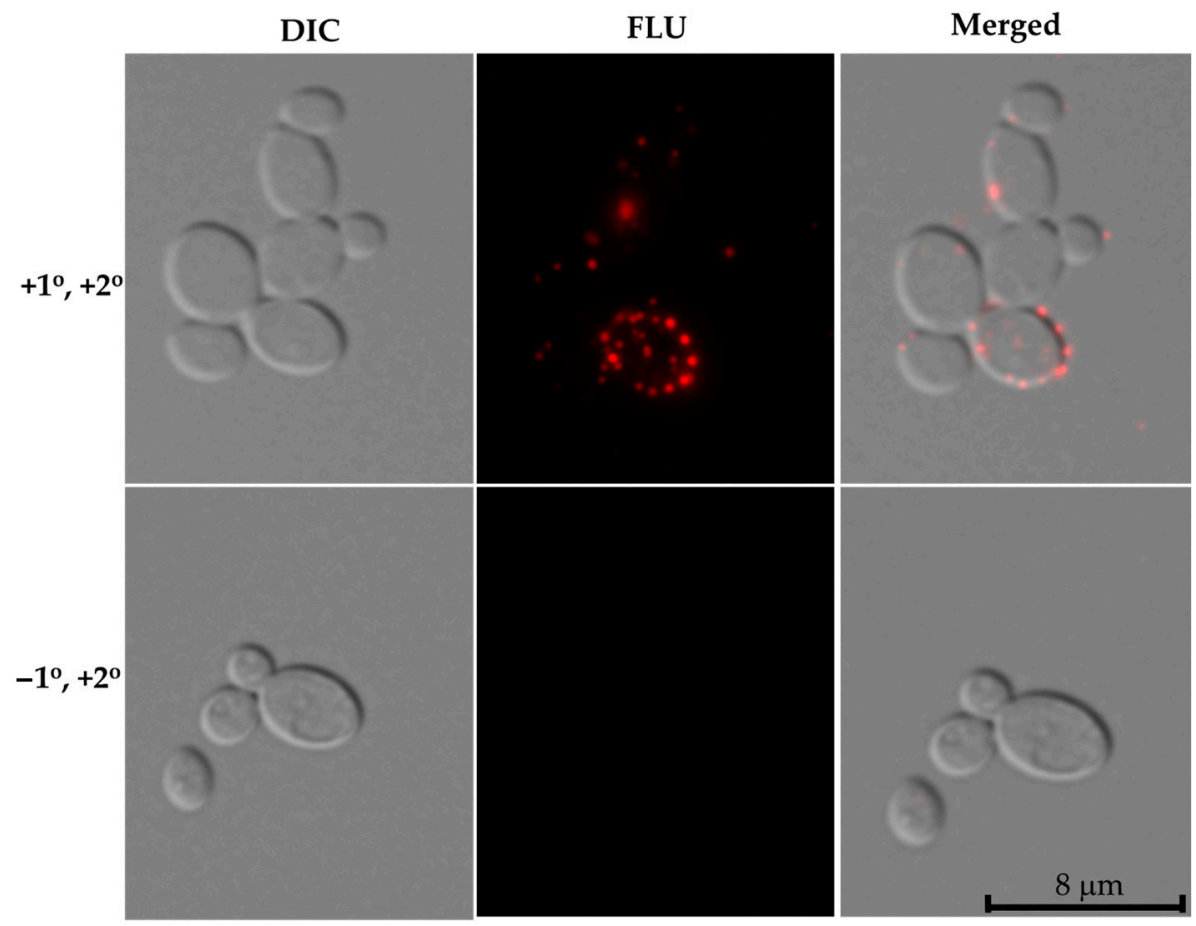

Figure 5. Immunofluorescence of cell surface expressed Nrg1p in C. auris. Synthetic sweat media grown $\mathrm{C}$. auris cells were stained with anti-CalNrg1 antibody followed by anti-rabbit Alexa Fluorsecondary antibody and observed under a fluorescence microscope (upper panel). Cells without primary antibody (anti-CalNrg1 antibody) and with anti-rabbit Alexa Fluor-secondary antibody are showed in lower panel (control). Scale bar $=8 \mu \mathrm{m}$.

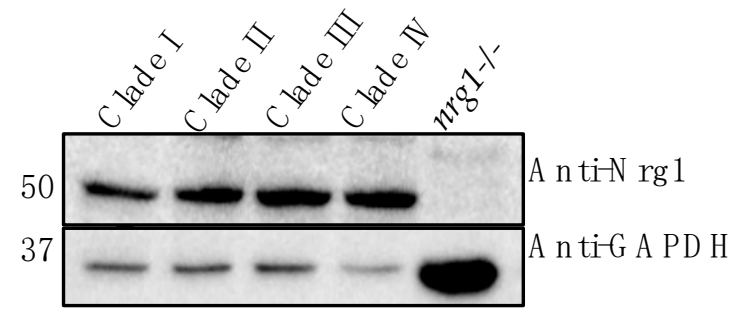

(a)

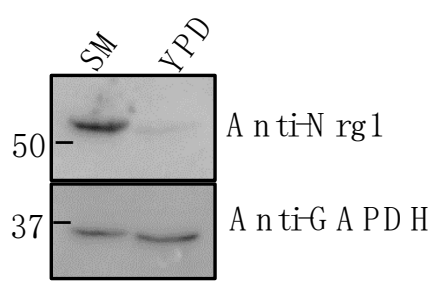

(b)

Figure 6. Nrg1p expression among different clades of $C$. auris and in different growth conditions. (a) Western blot of cytosolic proteins of 4 different clades of C. auris. Clade 1 (South Asia, Saudi Arabia, lab strain \#1126), Clade II (AR bank \#0381, East Asia), Clade III (AR bank \#0383, Africa), Clade IV (AR bank \#0386, South America). C. albicans $n r g 1^{-/-}$null mutant was included as a negative control. Anti-GAPDH (human, HRP-conjugate) was used as a loading control. (b) Cytosolic proteins from $C$. auris grown in synthetic sweat medium (SM) and YPD broth medium were probed against anti-CalNrg1 antibody (upper panel). Equal amounts of protein loading were confirmed by use of anti-GAPDH-HRP conjugate (human) antibody.

\subsection{Yeast-Hypha Regulation in C. auris Could Be Different from C. albicans}

C. albicans produces hyphae under hypha-inducing conditions (growth temperature at $37^{\circ} \mathrm{C}$, RPMI- 1640 medium and media containing serum, etc.) whereas C. auris remains in the yeast form under these conditions, hinting that its hyphal regulatory pathway(s) may be non-functional or different. However, C. auris forms elongated pseudohyphal phenotype when treated with a genotoxic compound, hydroxyurea (HU) [10], while the 
control cells (without HU) were mostly yeast cells which allowed us to determine whether C. auris Nrg1 plays any role in the pseudohyphal growth. Western analysis of cytosolic proteins from these two growth forms showed no differences in the expression levels of Nrg1p (Figure 7).

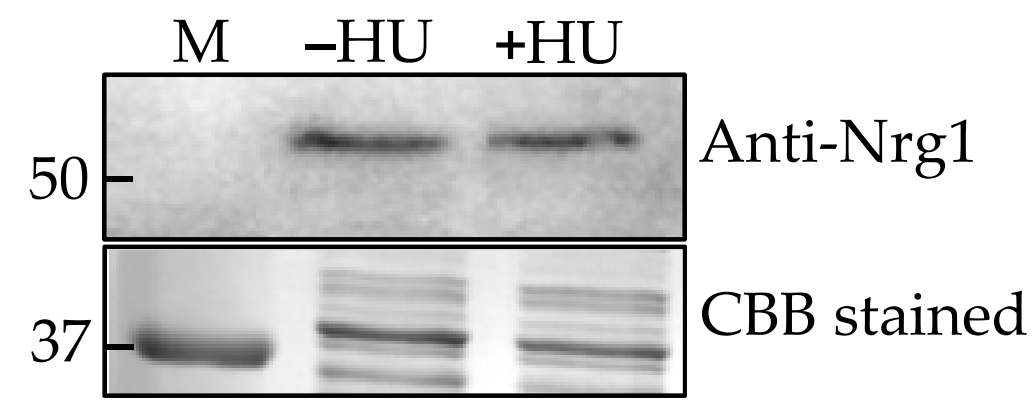

Figure 7. Nrg1p expression in C. auris treated with hydroxyurea (HU). Western analysis of cytosolic proteins from C. auris against CalNrg1 antibody (upper panel). Lane 1. Marker, Lane 2. Without HU, and Lane 3. With HU. SDS-PAGE gel of proteins from C. auris cells grown in sweat medium with and without HU followed by Coomassie brilliant blue (CBB) staining (lower panel).

\section{Discussion}

Candida auris, a newly emerged drug-resistant human fungal pathogen, was first reported in 2009 in Japan [33] and since then it has been isolated in over 40 countries across 6 continents [34]. C. auris is resistant to almost all clinical antifungal drugs and the CDC has listed this pathogen at an urgent threat level (fact sheet, CDC).

Although $C$. auris can be isolated from multiple sites in the body, it predominantly colonizes the skin and also persists in the environment, particularly in healthcare settings. Skin infections caused by C. auris in humans could be in a biofilm status. Despite the fact that $C$. auris produces fewer dense biofilms than $C$. albicans $[35,36], C$. auris can survive at high salt and other stress conditions [37] normally found in the skin niche and able to produce biofilms. Researchers have shown that $C$. auris is able to form 10-fold more biofilm than that of $C$. albicans in a skin-mimicking synthetic sweat medium that contains high salts and various lipids [15]. C. auris also has a high ability to colonize the skin, as demonstrated using porcine skin as an ex vivo model [15]. The high tolerance of this fungus to salty sweat medium mimicking skin conditions may play a role in its pathogenicity and persistence. We, therefore, as a first step, aimed to analyze the cell surface proteins from C. auris biofilms grown in the sweat medium.

Fungal cell surface proteins play a major role in pathogenesis. These proteins are one of the major groups of molecules that mediate the initial contact with its surrounding environment. Some cell surface proteins are covalently attached to mannan and other polysaccharides. Earlier studies have shown that the cell surface proteins can be extracted using an alkaline buffer containing $\beta$-mercaptoethanol (BME) $[19,20,38]$. By employing this method, we extracted a covalently attached cell surface and other soluble proteins from $C$. auris biofilms developed in sweat medium. As a control, we used planktonic cells grown in the same medium. Using 2-DE and silver staining, several differentially expressed proteins were detected from the BME extract of biofilm cells (Figure 1). This result shows that the $C$. auris biofilm produces some cell surface proteins at a higher level compared to planktonic cells. Although a limited number of protein spots were excised and analyzed by mass spectrometry, several of those detected are known to be found in the cell wall and/or on the cell surface.

Upregulated proteins that we identified by proteomics of biofilm cells include Spe3, Mdh1, Sod2, Ywp1 and Tdh3 (Table 2). Spe3p is involved in the biosynthesis of spermidine in C. albicans and also in many other fungi $[39,40]$. Spermidine is one of the three types of polyamines (putrescine and spermine are the others) that play a major role in various cellular processes including growth, oxidative and osmotic stress responses [39]. It is worth 
noting that $C$. auris hog1 mutant was acutely sensitive to organic oxidative- and osmoticstress-inducing agents [37]. Similarly, increased expression of Sod2p, the mitochondrial Mncontaining superoxide dismutase in C. auris biofilms, may provide antioxidant properties to the fungus against environmental or host oxidative stress. These proteins were shown to be overexpressed in C. albicans biofilms [41]. Certain proteins are involved in more than one cellular functions as in the case of C. glabrata Tdh3p that expressed more in biofilm than planktonic cells [42]. Proteomic analyses of cell surface proteins (BME) and extracellular vesicles of $C$. albicans yeast and hyphal morphologies were shown to contain Tdh3p $[43,44]$. Since Tdh3p is a moonlighting protein (involved in glycolysis, oxidoreductase activity and host-pathogen interaction), it may have some of these roles in C. auris biofilm growth. Future genetic studies (gene deletion or controlled expression) will determine the functional roles of these proteins in C. auris biofilm growth and virulence. C. albicans is known to cause biofilm-related infections and strains that are defective in hyphal growths fail to produce robust biofilms [30,31]. In contrast to the C. albicans biofilm production phenotype, C. auris produces ten-fold more biofilm without producing hyphae but with yeast cells [15]. This distinctive $C$. auris phenotype prompted us to search for a major negative regulator(s) of hyphal growth in $C$. auris. In conjunction with this notion, we have also identified some zinc-binding proteins in our 2-DE mass spectometry analyses. Nrg1p is a zinc-binding transcription factor that regulates hyphal growth negatively in co-operation with Tup1p in C. albicans [11], and the role and expression levels of Nrg1p in C. auris is unknown, although an ortholog is present. Further, Tup1, a zinc transcription factor similar to Nrg1p, has no hyphal regulatory function in C. auris [10].

Using an anti-Nrg1 antibody, we showed that Nrg1p is present in the cell surface extracts from both types of cells (Figure $2 b$ ). To confirm that the cell surface proteins are not contaminated with the cytosolic proteins during BME extraction, we probed these proteins with an anti-GAPDH (human) antibody that reacts only to the cytosolic proteins but not to the cell surface proteins (Figure 2b, lower panel). The presence of Nrg1p in the BME extract of $C$. auris biofilm cells was further demonstrated by a 2-DE resolved Western blot (Figure $4 \mathrm{~b}$ ). Interestingly, the immunofluorescence assay clearly showed the presence of Nrg1p on the cell surfaces of C. auris in vivo (Figure 5). Taken together, a zinc transcription factor Nrg1p, normally found in the nucleus, can also be present on the cell surface of $C$. auris cells. However, the mechanism of Nrg1p transport to the cell surface and its function(s) on the cell surface remain to be determined.

Structural data for fungal Nrg1p are not available. However, the three-dimensional prediction of Nrg1 proteins from C. auris and C. albicans by the I-TASSER program and their structural alignment by RaptorX programs rendered a superimposed model showing Nrg1 proteins from both species have a similar fold (Figure $3 b-d$ ). Importantly, the zinc-binding and the DNA-binding C-terminal regions are highly conserved and similar in structure, including the alpha-helices and $ß$-sheet folds of the DNA binding regions of the Class-I zinc-finger transcription factors (Figure 3b-d) [32]. It is unclear if the lack of 44 residues at the C-terminus of Nrg1 in C. auris may have functional implications.

It is reasonable to speculate that the hyphal regulatory role of Nrg1p in $C$. auris may be different or absent from C. albicans Nrg1p. For example, C. albicans hyphae and pseudohyphae were shown to convert into yeast cells when they were treated with hyphal growth inhibitors called gymnemic acids (GAs) [45]. However, when pseudohyphae of $C$. auris were treated with GAs, they did not revert to yeasts and they remain pseudohyphae. Further, the expression level of Nrg1p in yeast and pseudohyphal growth forms of C. auris without and with HU, respectively, did not show any differences between the two growth forms (Figure 7) which may suggest that Nrg1p may have a limited role in the yeast-hyphal morphogenesis in C. auris. Alternatively, the growth condition or host niche-dependent cues may regulate hyphal morphogenesis in this fungus. When C. auris was grown in YPD with $10 \% \mathrm{NaCl}$, elongated pseudohyphal-like cells were formed [46]. Similarly, C. auris can filament when it is passaged in the mammalian body [9]. It is also worth mentioning that the representative strains from all four clades (Clade I-IV) that we analyzed contain Nrg1p 
(Figure 6a). Thus, C. auris is more tolerant to various stress conditions when compared to other Candida species which might help in the unique lifestyle of $C$. auris.

In conclusion, $C$. auris produces several differentially expressed cell surface proteins during its biofilm growth. Despite its predominant yeast form of growth, C. auris produces robust biofilm, and the $\mathrm{Nrg} 1$ ortholog is expressed on the cell surface of C. auris. While the function of $\mathrm{Nrg} 1 \mathrm{p}$ is currently unknown, representative strains from all four known clades of C. auris express Nrg1p.

Supplementary Materials: The following are available online at https:/ /www.mdpi.com/article/ $10.3390 /$ jof7040262/s1, Figure S1. Determination of specificity of the anti-CalNrg1 antibody. The CalNrg1 antibody was divided into two equal aliquots. One of the aliquots was incubated to a PVDF-membrane containing the immobilized C. albicans purified Nrg1 protein overnight at $4{ }^{\circ} \mathrm{C}$ on a roller. To verify if the $C$. albicans $\mathrm{Nrg} 1 \mathrm{p}$ depleted antibody can still detect $C$. auris $\mathrm{Nrg} 1 \mathrm{p}$, one set of cytosolic proteins from C. auris (clades I \& II, Table 1) was analyzed by Western blot (left panel). An identical blot was analyzed in parallel with the original (undepleted) anti-Nrg1 antibody (right panel). C. albicans nrg1 null mutant was also included as a negative control. While the undepleted anti-Nrg1 antibody reacted to a single protein band of about $52 \mathrm{kDa}$, the depleted antibody failed to show a strong reaction to C. auris cellular proteins suggesting the anti-Cal $\mathrm{Nrg} 1$ antibody recognizes $\mathrm{Nrg} 1 \mathrm{p}$ specifically. The anti-GAPDH HRP-conjugate (human) antibody was used as a loading control.

Author Contributions: Conceptualization, supervision, project administration and funding acquisition, G.V.; methodology, data analysis, validation and writing, review and editing, A.P. and G.V. Both authors have read and agreed to the published version of the manuscript.

Funding: This research was supported by the Division of Biology, Kansas State University (KSU) the Startup Research funds and Johnson Cancer Research Center's (JCRC) Innovative Research Award, KSU, to G.V., and the JCRC's Summer Research stipend to A.P.

Institutional Review Board Statement: Not applicable.

Informed Consent Statement: Not applicable.

Data Availability Statement: Not applicable. (No gene expression or genomic data are involved).

Acknowledgments: We thank the Centers for Disease Control and Prevention (CDC) and the Food and Drug Administration (FDA) Antibiotic Resistance (AR) isolate bank for providing C. auris isolates. Mass spectrometry analyses were performed at the Center for Genomics and Proteomics at Oklahoma State University. We also thank Ruth Welti, Division of Biology, KSU, for critical reading of the manuscript.

Conflicts of Interest: The authors declare no conflict of interest. The funders had no role in the design of the study; in the collection, analyses, or interpretation of data; in the writing of the manuscript, or in the decision to publish the results.

\section{References}

1. Krüger, W.; Vielreicher, S.; Kapitan, M.; Jacobsen, I.D.; Niemiec, M.J. Fungal-Bacterial Interactions in Health and Disease. Pathogens 2019, 8, 70. [CrossRef] [PubMed]

2. Lockhart, S.R.; Etienne, K.A.; Vallabhaneni, S.; Farooqi, J.; Chowdhary, A.; Govender, N.P.; Colombo, A.L.; Calvo, B.; Cuomo, C.A.; Desjardins, C.A.; et al. Simultaneous Emergence of Multidrug-Resistant Candida auris on 3 Continents Confirmed by Whole-Genome Sequencing and Epidemiological Analyses. Clin. Infect Dis. 2017, 64, 134-140. [CrossRef]

3. Iguchi, S.; Itakura, Y.; Yoshida, A.; Kamada, K.; Mizushima, R.; Arai, Y.; Uzawa, Y.; Kikuchi, K. Candida auris: A pathogen difficult to identify, treat, and eradicate and its characteristics in Japanese strains. J. Infect. Chemother. 2019, 25, 743-749. [CrossRef]

4. Adams, E.; Quinn, M.; Tsay, S.; Poirot, E.; Chaturvedi, S.; Southwick, K.; Greenko, J.; Fernandez, R.; Kallen, A.; Vallabhaneni, S.; et al. Candida auris in Healthcare Facilities, New York, USA, 2013-2017. Emerg. Infect. Dis. 2018, 24, 1816-1824. [CrossRef]

5. Chowdhary, A.; Sharma, C.; Duggal, S.; Agarwal, K.; Prakash, A.; Singh, P.K. New clonal strain of Candida auris, Delhi, India. Emerg. Infect. Dis. 2013, 19, 1670-1673. [CrossRef] [PubMed]

6. Calvo, B.; Melo, A.S.; Perozo-Mena, A.; Hernandez, M.; Francisco, E.C.; Hagen, F.; Meis, J.F.; Colombo, A.L. First report of Candida auris in America: Clinical and microbiological aspects of 18 episodes of candidemia. J. Infect. 2016, 73, 369-374. [CrossRef] [PubMed] 
7. Chakrabarti, A.; Sood, P.; Rudramurthy, S.M.; Chen, S.; Kaur, H.; Capoor, M.; Chhina, D.; Rao, R.; Eshwara, V.K.; Xess, I.; et al. Incidence, characteristics and outcome of ICU-acquired candidemia in India. Intensiv. Care Med. 2014, 41, 285-295. [CrossRef] [PubMed]

8. Borman, A.M.; Szekely, A.; Johnson, E.M. Comparative Pathogenicity of United Kingdom Isolates of the Emerging Pathogen Candida auris and Other Key Pathogenic Candida Species. mSphere 2016, 1, e00189-16. [CrossRef]

9. Yue, H.; Bing, J.; Zheng, Q.; Zhang, Y.; Hu, T.; Du, H.; Wang, H.; Huang, G. Filamentation in Candida auris, an emerging fungal pathogen of humans: Passage through the mammalian body induces a heritable phenotypic switch. Emerg. Microbes Infect. 2018, 7, 188. [CrossRef]

10. Bravo Ruiz, G.; Ross, Z.K.; Gow, N.A.R.; Lorenz, A. Pseudohyphal Growth of the Emerging Pathogen Candida auris Is Triggered by Genotoxic Stress through the S Phase Checkpoint. mSphere 2020, 5. [CrossRef] [PubMed]

11. Braun, B.R.; Kadosh, D.; Johnson, A.D. NRG1, a repressor of filamentous growth in Candida albicans, is down-regulated during filament induction. EMBO J. 2001, 20, 4753-4761. [CrossRef]

12. Murad, A.A.; Leng, P.; Straffon, M.; Wishart, J.; Macaskill, S.; Maccallum, D.M.; Schnell, N.; Talibi, D.; Marechal, D.; Tekaia, F.; et al. NRG1 represses yeast-hypha morphogenesis and hypha-specific gene expression in Candida albicans. EMBO J. 2001, 20, 4742-4752. [CrossRef]

13. Uppuluri, P.; Pierce, C.G.; Thomas, D.P.; Bubeck, S.S.; Saville, S.P.; Lopez-Ribot, J.L. The Transcriptional Regulator Nrg1p Controls Candida albicans Biofilm Formation and Dispersion. Eukaryot. Cell 2010, 9, 1531-1537. [CrossRef]

14. Saville, S.P.; Lazzell, A.L.; Monteagudo, C.; Lopez-Ribot, J.L. Engineered Control of Cell Morphology in vivo Reveals Distinct Roles for Yeast and Filamentous Forms of Candida albicans during Infection. Eukaryot. Cell 2003, 2, 1053-1060. [CrossRef]

15. Horton, M.V.; Johnson, C.J.; Kernien, J.F.; Patel, T.D.; Lam, B.C.; Cheong, J.Z.A.; Meudt, J.J.; Shanmuganayagam, D.; Kalan, L.R.; Nett, J.E. Candida auris Forms High-Burden Biofilms in Skin Niche Conditions and on Porcine Skin. mSphere 2020, 5. [CrossRef]

16. Abdalhamid, B.; Almaghrabi, R.; Althawadi, S.; Omrani, A. First report of Candida auris infections from Saudi Arabia. J. Infect. Public Health 2018, 11, 598-599. [CrossRef]

17. Gillum, A.M.; Tsay, E.Y.H.; Kirsch, D.R. Isolation of the Candida albicans gene for orotidine-5'-phosphate decarboxylase by complementation of S. cerevisiae ura3 and E. coli pyrF mutations. Mol. Genet. Genom. 1984, 198, 179-182. [CrossRef]

18. Noble, S.M.; French, S.; Kohn, L.A.; Chen, V.; Johnson, A.D. Systematic screens of a Candida albicans homozygous deletion library decouple morphogenetic switching and pathogenicity. Nat. Genet. 2010, 42, 590-598. [CrossRef] [PubMed]

19. Casanova, M.; Chaffin, W.L. Cell wall glycoproteins of Candida albicans as released by different methods. J. Gen. Microbiol. 1991, 137, 1045-1051. [PubMed]

20. Vediyappan, G.; Bikandi, J.; Braley, R.; Chaffin, W.L. Cell surface proteins of Candida albicans: Preparation of extracts and improved detection of proteins. Electrophoresis 2000, 21, 956-961. [CrossRef]

21. Bradford, M.M. A rapid and sensitive method for the quantitation of microgram quantities of protein utilizing the principle of protein-dye binding. Anal. Biochem. 1976, 72, 248-254. [CrossRef]

22. Roy, A.; Kucukural, A.; Zhang, Y. I-TASSER: A unified platform for automated protein structure and function prediction. Nat. Protoc. 2010, 5, 725-738. [CrossRef] [PubMed]

23. Peng, J.; Xu, J. Raptorx: Exploiting structure information for protein alignment by statistical inference. Proteins Struct. Funct. Bioinform. 2011, 79, 161-171. [CrossRef] [PubMed]

24. Pemberton, L.F. Preparation of Yeast Cells for Live-Cell Imaging and Indirect Immunofluorescence. Adv. Struct. Saf. Stud. 2014, 1205, 79-90.

25. Bonhomme, J.; Chauvel, M.; Goyard, S.; Roux, P.; Rossignol, T.; D’Enfert, C. Contribution of the glycolytic flux and hypoxia adaptation to efficient biofilm formation by Candida albicans. Mol. Microbiol. 2011, 80, 995-1013. [CrossRef]

26. Hwang, C.S.; Rhie, G.; Kim, S.T.; Kim, Y.R.; Huh, W.K.; Baek, Y.U.; Kang, S.O. Copper- and zinc-containing superoxide dismutase and its gene from Candida albicans. Biochim. Biophys. Acta 1999, 1427, 245-255. [CrossRef]

27. Rhie, G.; Hwang, C.S.; Brady, M.; Kim, S.T.; Kim, Y.R.; Huh, W.K.; Baek, Y.U.; Lee, B.H.; Lee, J.S.; Kang, S.O. Manganese-containing superoxide dismutase and its gene from Candida albicans. Biochim. Biophys. Acta 1999, 1426, 409-419. [CrossRef]

28. Granger, B.L.; Flenniken, M.L.; Davis, D.A.; Mitchell, A.P.; Cutler, J.E. Yeast wall protein 1 of Candida albicans. Microbiology 2005, 151, 1631-1644. [CrossRef] [PubMed]

29. Seneviratne, C.J.; Wang, Y.; Jin, L.; Abiko, Y.; Samaranayake, L.P. Candida albicans biofilm formation is associated with increased anti-oxidative capacities. Proteomics 2008, 8, 2936-2947. [CrossRef]

30. Mitchell, K.F.; Zarnowski, R.; Andes, D.R. Fungal Super Glue: The Biofilm Matrix and Its Composition, Assembly, and Functions. PLoS Pathog. 2016, 12, e1005828. [CrossRef]

31. Richard, M.L.; Nobile, C.J.; Bruno, V.M.; Mitchell, A.P. Candida albicans Biofilm-Defective Mutants. Eukaryot. Cell 2005, 4, 1493-1502. [CrossRef]

32. MacPherson, S.; LaRochelle, M.; Turcotte, B. A Fungal Family of Transcriptional Regulators: The Zinc Cluster Proteins. Microbiol. Mol. Biol. Rev. 2006, 70, 583-604. [CrossRef]

33. Satoh, K.; Makimura, K.; Hasumi, Y.; Nishiyama, Y.; Uchida, K.; Yamaguchi, H. Candida auris sp. nov., a novel ascomycetous yeast isolated from the external ear canal of an inpatient in a Japanese hospital. Microbiol. Immunol. 2009, 53, 41-44. [CrossRef]

34. Du, H.; Bing, J.; Hu, T.; Ennis, C.L.; Nobile, C.J.; Huang, G. Candida auris: Epidemiology, biology, antifungal resistance, and virulence. PLoS Pathog. 2020, 16, e1008921. [CrossRef] 
35. Sherry, L.; Ramage, G.; Kean, R.; Borman, A.; Johnson, E.M.; Richardson, M.D.; Rautemaa-Richardson, R. Biofilm-Forming Capability of Highly Virulent, Multidrug-Resistant Candida auris. Emerg. Infect. Dis. 2017, 23, 328-331. [CrossRef]

36. Larkin, E.; Hager, C.; Chandra, J.; Mukherjee, P.K.; Retuerto, M.; Salem, I.; Long, L.; Isham, N.; Kovanda, L.; Borroto-Esoda, K.; et al. The Emerging Pathogen Candida auris: Growth Phenotype, Virulence Factors, Activity of Antifungals, and Effect of SCY-078, a Novel Glucan Synthesis Inhibitor, on Growth Morphology and Biofilm Formation. Antimicrob. Agents Chemother. $2017,61$. [CrossRef]

37. Day, A.M.; McNiff, M.M.; Dantas, A.D.S.; Gow, N.A.R.; Quinn, J. Hog1 Regulates Stress Tolerance and Virulence in the Emerging Fungal Pathogen Candida auris. mSphere 2018, 3, e00506-18. [CrossRef] [PubMed]

38. Chaffin, W.L. Candida albicans cell wall proteins. Microbiol. Mol. Biol. Rev. 2008, 72, 495-544. [CrossRef] [PubMed]

39. Valdés-Santiago, L.; Ruiz-Herrera, J. Stress and polyamine metabolism in fungi. Front. Chem. 2014, 1, 42. [CrossRef] [PubMed]

40. Rocha, R.O.; Wilson, R.A. Essential, deadly, enigmatic: Polyamine metabolism and roles in fungal cells. Fungal Biol. Rev. 2019, 33, 47-57. [CrossRef]

41. Martínez-Gomariz, M.; Perumal, P.; Mekala, S.; Nombela, C.; Chaffin, W.L.; Gil, C. Proteomic analysis of cytoplasmic and surface proteins from yeast cells, hyphae, and biofilms of Candida albicans. Proteomics 2009, 9, 2230-2252. [CrossRef] [PubMed]

42. Seneviratne, C.J.; Wang, Y.; Jin, L.; Abiko, Y.; Samaranayake, L.P. Proteomics of drug resistance in Candida glabrata biofilms. Proteomics 2010, 10, 1444-1454. [CrossRef] [PubMed]

43. Gil-Bona, A.; Parra-Giraldo, C.M.; Hernáez, M.L.; Reales-Calderon, J.A.; Solis, N.V.; Filler, S.G.; Monteoliva, L.; Gil, C. Candida albicans cell shaving uncovers new proteins involved in cell wall integrity, yeast to hypha transition, stress response and host-pathogen interaction. J. Proteom. 2015, 127, 340-351. [CrossRef]

44. Gil-Bona, A.; Amador-García, A.; Gil, C.; Monteoliva, L. The external face of Candida albicans: A proteomic view of the cell surface and the extracellular environment. J. Proteom. 2018, 180, 70-79. [CrossRef] [PubMed]

45. Vediyappan, G.; Dumontet, V.; Pelissier, F.; D’Enfert, C. Gymnemic Acids Inhibit Hyphal Growth and Virulence in Candida albicans. PLoS ONE 2013, 8, e74189. [CrossRef] [PubMed]

46. Wang, X.; Bing, J.; Zheng, Q.; Zhang, F.; Liu, J.; Yue, H.; Tao, L.; Du, H.; Wang, Y.; Wang, H.; et al. The first isolate of Candida auris in China: Clinical and biological aspects. Emerg. Microbes Infect. 2018, 7, 93. [CrossRef] 